\title{
Thematic mapping with a twist
}

\author{
Kenneth Field \\ EsriInc,kfield@esri.com
}

Keywords: cartography, map design, data visualisation

\begin{abstract}
:
After spending many years making countless thematic maps of UK and US elections I had many people quiz me on what I would do for the 2020 US presidential election. I'd just finished writing my second book, Thematic Mapping, in which I used the 2016 US Presidentical election to make 101 maps of the same data. The whole point of that book is to demonstrate the breadth and depth of cartographic representation and how the thematic map choice helps determine the way in which a reader interprets the patterns inherent in the data being mapped. Yet for the 2020 election people seemed to want more than a choropleth; and more than the examples I'd designed in the book; something different.
\end{abstract}

Doing something different is always the challenge with thematic mapping. But why different? Why not just a solid, well known technique done well? People seem to want 'different' and to see how we can stretch the boundaries of cartography. They want something visually striking.

Working from home during the coronavirus pandemic afforded me that opportunity as I literally constructed a new kind of multivariate 3D thematic map from wood, screws, paint, glue and epoxy resin. It's a physical model of the results of the 2020 election with an inlay basemap of results with a bas-relief grid of screws representing population density and margin of victory through gauge, height, and colour (Figure 1). For good measure I also created a 3D digital twin which acted as both a blueprint, a testbed, a data source (for CNC routers), and a map in its own right.

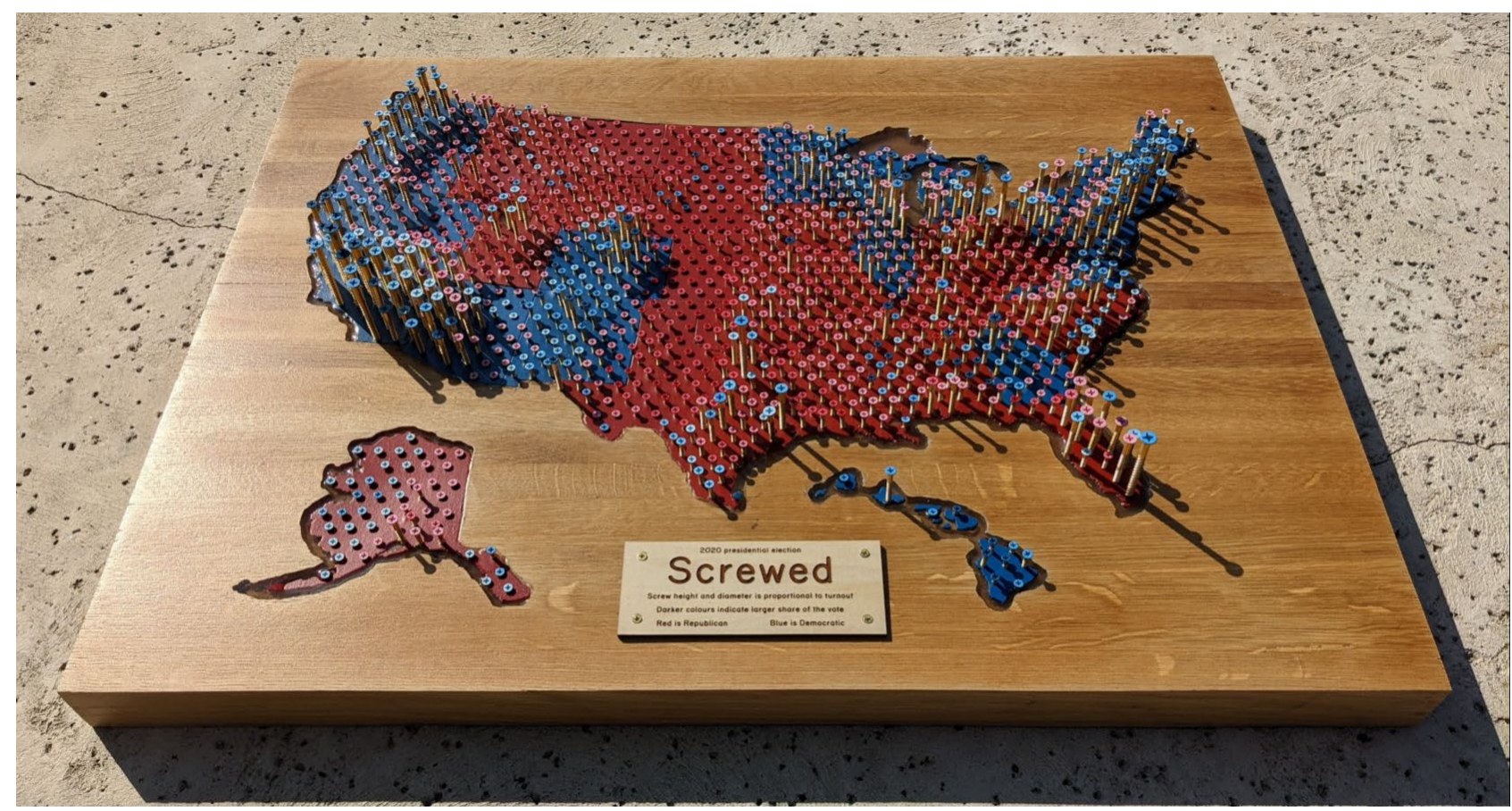

Figure 1: Screwed: 2020 Presidential election

This talk will explore the ideas, approach, decisions, risk, and catharsis (living in the US during both Trump and the COVID-19 pandemic!) of building a physical map using non-conventional cartographic tools such as circular saws, laser cutters, routers, and sanders. I'll also show how the digital twin can be built in an interactive 3D map using more conventional mapping tools. Along the way I'll signpost some of the more general benefits and drawbacks of using 3D to make thematic maps.

I also created a second map during lockdown between March 2020 and March 2021 which I'll discuss. I captured GPS tracklogs while walking my dog morning and evening; building a database of 730 walking routes. I wanted to experiment 
with the challenge of creating maps of a lot of coincident data, in this case overprinted lines. This part of the talk will explore some of those choices, and expose what I think are best practices for this cartographic dilemma. The map also ended up becoming an animation since that allowed me to incorporate the temporal dimension of the walks. There were some interesting patterns in the data and show how our dog was challenged by the heat of the Southern Californian summer.

I might even talk about a map my dog, Wisley, helped me make to keep me sane. 